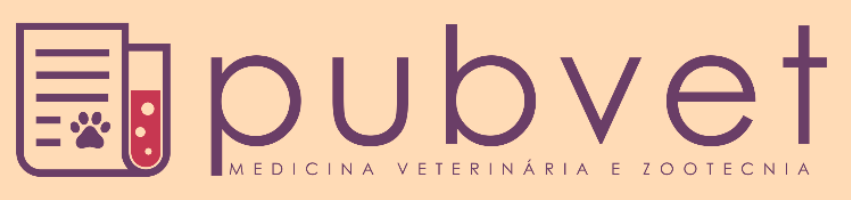

https://doi.org/10.31533/pubvet.v14n8a638.1-7

\title{
Fatores predisponentes e sua influência no bem-estar de cães obesos
}

\author{
Diely Sonvez Claudino da Silva ${ }^{1 *} \bullet$, Vanessa Yuri de Lima ${ }^{2} \bullet$ \\ ${ }^{1}$ Discente do curso de graduação em Medicina Veterinária da Pontifícia Universidade Católica do Paraná, Toledo, Paraná, Brasil \\ ${ }^{2}$ Docente do curso de graduação em Medicina Veterinária da Pontifícia Universidade Católica do Paraná, Toledo, Paraná, Brasil. \\ *Autor para correspondência, E-mail: dielysonvez@hotmail.com
}

Resumo. A obesidade pode ser tratada como uma condição patológica caracterizada por um acúmulo de gordura maior que o necessário para otimização das funções do corpo, suficiente para deteriorá-las e prejudicar a boa saúde e o bem-estar animal. Um cão obeso apresenta um acúmulo de gordura responsável pelo aumento do peso em pelo menos $15 \mathrm{a}$ $20 \%$, em relação ao seu peso ideal. A obesidade é causada por um consumo excessivo de calorias que excedem às necessidades energéticas, embora fatores como genética e algumas condições patológicas secundárias possam estar envolvidas na etiologia. Quando um cão está excessivamente obeso, aumentam os problemas circulatórios, articulares e locomotores, além de enfermidades de pele e tumores. Atualmente, a obesidade acomete cerca de $30 \%$ dos cães, sendo, portanto, reconhecida como uma doença grave, com múltiplas consequências sobre a saúde do animal e não apenas um problema estético. Objetivou-se neste trabalho discutir sobre os efeitos da obesidade em cães. Participaram da pesquisa 23 tutores com idade entre 18 e 60 anos, variando o grau de instrução e renda familiar mensal. Estes eram tutores de 25 cães, castrados e inteiros. Cães de meia idade apresentavam maiores densidade corpóreas, justamente porque o tutor fornecia alimentos (rações, comidas em geral) e petiscos à vontade, e porque esses animais não praticavam exercícios regulares. Os tutores apresentaram dificuldade em perceber a condição corpórea do seu cão. Algumas práticas devem ser consideradas, como eliminar os maus hábitos (refeições diárias excessivas e atividade física insuficiente) e avaliar as necessidades energéticas. Assim sendo, é possível atingir um peso corporal saudável.

Palavras chave: cães, obesidade, patologias, práticas

\section{Predisposing factors and their influence on the welfare of obese dogs}

Abstract. The obesity can be treated as a pathological condition characterized by an accumulation of fat greater than that necessary for the optimization of body functions, sufficient to deteriorate them and impair good health and animal welfare. An obese dog has an accumulation of fat responsible for increasing weight by at least 15 to $20 \%$, in relation to its ideal weight. Obesity is caused by an excessive consumption of calories that exceed energy needs, although factors such as genetics and some secondary pathological conditions may be involved in the etiology. When a dog is excessively obese, circulatory, joint and locomotor problems increase, in addition to skin diseases and tumors. Currently, obesity affects about $30 \%$ of dogs and is, therefore, recognized as a serious disease, with multiple consequences on the health of the animal and not just an aesthetic problem. Some practices must be considered, such as eliminating bad habits (excessive daily meals and insufficient physical activity), assessing energy needs, weighing and examining your dog in order to achieve a healthy body weight. The objective of this study was to discuss the effects of obesity in dogs. 23 tutors aged between 18 and 60 years participated in the research, varying the level of education and monthly family income. These were tutors of $25 \mathrm{dogs}$, neutered and whole. Middle-aged dogs had higher body density, precisely because 
the tutor provided food (rations, food in general) and snacks at will, and because these animals did not practice regular exercises. The tutors found it difficult to perceive their dog's body condition. Some practices must be considered, such as eliminating bad habits (excessive daily meals and insufficient physical activity) and assessing energy needs. Therefore, it is possible to achieve a healthy body weight.

Keywords: dogs, obesity, pathologies, practices

\section{Factores predisponentes y su influencia en el bienestar de los perros obesos}

Resumen. La obesidad puede tratarse como una patología caracterizada por acumulo de grasa, mayor que lo necesario para la optimización de las funciones del organismo, ocasionando daño y perjudicando la salud y bienestar animal. Un perro obeso tiene una acumulación de grasa responsable de aumentar el peso al menos entre un 15 y un $20 \%$, en relación con su peso ideal. La obesidad se produce por un consumo excesivo de calorías que superan las necesidades energéticas, aunque en la etiología pueden intervenir factores como la genética y algunas patologías secundarias. Cuando un perro es excesivamente obeso aumentan los problemas circulatorios, articulares y locomotores, además de las enfermedades cutáneas y los tumores. Actualmente, la obesidad afecta a alrededor del $30 \%$ de los perros, por lo que está reconocida como una enfermedad grave, con múltiples consecuencias en la salud del animal y no solo un problema estético. El objetivo de este estudio fue discutir los efectos de la obesidad en perros. Participaron de la investigación 23 tutores con edades comprendidas entre los 18 y los 60 años, variando el nivel educativo y el ingreso familiar mensual. Estos eran los guardianes de 25 perros, castrados y enteros. Los perros de mediana edad tenían una mayor densidad corporal, precisamente porque el tutor proporcionaba alimentos (raciones, comida en general) y bocadillos a voluntad, y porque estos animales no practicaban ejercicios regulares. A los tutores les resultó difícil percibir la condición corporal de su perro. Hay que tener en cuenta algunas prácticas, como eliminar los malos hábitos (comidas diarias excesivas y actividad física insuficiente) y evaluar las necesidades energéticas. Por tanto, es posible alcanzar un peso corporal saludable.

Palabras clave: perros, obesidad, patologías, prácticas

\section{Introdução}

A obesidade trata-se de uma condição patológica caracterizada por deteriorar e prejudicar a boa saúde e o bem-estar animal, já que é representada pelo acúmulo de gordura maior que o necessário para otimização das funções do corpo (Biourges, 1997; Morgante, 1999). É importante ressaltar que a obesidade é ocasionada por um consumo excessivo de calorias que excedem o limiar das necessidades energéticas, embora fatores como genética e algumas condições patológicas secundárias possam estar envolvidas na etiologia (Fleeman et al., 2006). Problemas circulatórios, articulares e locomotores, além de doenças de pele e tumores podem aumentar quando um cão está excessivamente obeso (Zoran, 2010).

Em cães, ocorre consequente acúmulo excessivo de gordura corporal quando o peso do animal está pelo menos 15\% acima do ideal (Burkholder \& Toll, 2000). A obesidade pode elevar o risco de aparecimento de várias complicações (Diez \& Nguyen, 2006), além disso, pode resultar em redução da sua qualidade de vida e da longevidade, tornando-se prejudicial à saúde do cão (Laflamme, 2006; Zoran, 2010). O aumento excessivo do peso corporal é uma enfermidade universal de prevalência crescente, sendo um dos principais problemas de saúde pública nos tempos atuais (Mancini, 2001). A manutenção do peso corporal ideal é fator determinante para conservação da saúde, bem-estar e qualidade de vida. Em situações em que ocorrem desbalanços nutricionais e energéticos, como, por exemplo, o desequilíbrio entre a ingestão calórica e a diminuição da prática da atividade física ocorre a obesidade ( $\underline{\text { Salve, 2006) }}$.

Segundo Feitosa et al. (2015), entre 20 a $40 \%$ da população canina são acometidas pela obesidade. O intervalo de idade de maior prevalência se situa de 5 a 10 anos (Carciofi, 2005) e pode estar relacionada à diminuição do gasto energético, reduzidas atividades físicas e alterações no metabolismo corporal em função da idade (Lazzarotto, 1999). A obesidade é capaz de aumentar a incidência de algumas doenças (Brunetto et 
al., 2011). Esse conceito deixa implícito um transtorno prejudicial para a saúde do indivíduo. Este fato, unido à elevada frequência com que se observa a afecção, faz da obesidade uma das patologias mais relevantes de má nutrição na prática clínica de pequenos animais (Vigoureux, 2014).

Algumas doenças podem estar envolvidas no acúmulo de peso, como o hipotireoidismo e o hiperadrenocorticismo, também o uso indiscriminado de fármacos. Entretanto, mesmo com todos esses fatores, a maior causa do desenvolvimento da obesidade em cães se dá pelo descompasso positivo entre o consumo de energia e o seu gasto diário. Portanto, práticas errôneas como o fator alimentar, a suscetibilidade genética, o exercício físico desregrado, o manejo errôneo do proprietário e o descompasso nutricional tem grande importância quando se fala em obesidade e pode aumentar relativamente esse distúrbio do peso corpóreo (German, 2006). Nota-se, também, a influência da elaboração dos alimentos quando se fala em combate a obesidade e bem-estar. Assim, um alimento corretamente balanceado e pensado em cada especificidade, proporciona bem-estar, e combate possíveis riscos de sobrepeso ao cão. Há tendência de produzir alimentos específicos para cada animal, por exemplo, a comida úmida para animais idosos, cumpre sua demanda energética com ótima palatabilidade. Tais especificidades são importantes não somente para cães idosos, mas também para filhotes, animais imunossuprimidos, deficientes renais e hepáticos.

Atualmente, os animais vivem em casas e apartamentos, não caçam e não se exercitam como quando tinham vida livre. Essa mudança no estilo de vida contribuiu significativamente para o aumento dos índices da obesidade canina (Rodrigues, 2011).

A castração é outro importante fator de risco para a obesidade em cães, devido à redução na taxa metabólica (Oliveira et al., 2010). Segundo Silva (2014), fêmeas castradas têm maior predisposição à obesidade, isso por terem a ausência de hormônios sexuais. Já os machos castrados, podem apresentar uma diminuição de estímulos sexuais, e consequentemente, redução da sua atividade física pelo fato do estrógeno exercer um efeito inibitório no apetite. $\mathrm{O}$ maior consumo de alimentos também está relacionado com a ausência do efeito de hormônios gonadotróficos.

A prevenção da obesidade em animais domésticos deve priorizar o bem-estar animal, visando principalmente à reeducação do tutor. Quando se compara animais hígidos e obesos, a qualidade de vida e a incidência de doenças são índices significativos (Armstrong et al., 2008). A obesidade traz inúmeras consequências, mas dificilmente o proprietário é capaz de diagnosticar. Isso gera uma deterioração das capacidades normais do animal que passa a ter seus movimentos reduzidos e suas capacidades fisiológicas diminuídas, sendo incapaz de expressar suas necessidades, o que fere fortemente o princípio de bem-estar animal (Guimarães \& Tudury, 2006).

A atual pesquisa foi realizada com tutores de cães que frequentaram a Clínica Veterinária (Clivet) da Pontifícia Universidade Católica do Paraná (PUCPR), Campus Toledo, no período de agosto de 2018 a junho de 2019, e teve como finalidade a realização de uma entrevista, no intuito de verificar sua percepção relacionada ao seu animal de estimação quando se trata de peso ideal, levando em consideração à sensibilidade de raças caninas à obesidade e seu Escore de Condição Corporal.

\section{Material e métodos}

\section{Animais e ambiente}

Os cães que estavam em consulta de rotina, a partir da sua classificação, se considerados obesos, tiveram a participação de seus tutores. Para tanto, foram por avaliações como: idade, predisposição genética, sexo e castração, frequência no consumo de alimentos, nível de atividade voluntária e se fazia uso de algum medicamento e a susceptibilidade das raças caninas à obesidade. Foram obtidos também dados como: a classificação do Escore de Condição Corporal (Figura 1), na escola de 9 pontos, e o peso ideal para várias raças de cães.

\section{Entrevista com o tutor}

O tutor participante da pesquisa respondeu voluntariamente as perguntas, após ter assinado o Termo de Consentimento Livre e Esclarecido, aprovado pelo Comitê de Ética e Pesquisa da PUCPR (CAAE: 00764818.8.0000.0020), sobre o dia a dia do seu cão e informou sobre seus cuidados e proteção com o animal. 
Confirmado a obesidade do cão pelas avaliações, o tutor foi informado sobre a situação atual do seu animal e sobre possíveis mudanças no seu dia a dia, incluindo a prática de atividades físicas diárias, a fim de se alcançar o bem-estar. O tutor compreendeu que a obesidade se trata de uma doença, e que pode trazer consequências para saúde do seu animal. É de suma importância a colaboração dos tutores e das outras pessoas que convivem com o cão para o processo de redução de peso do animal.

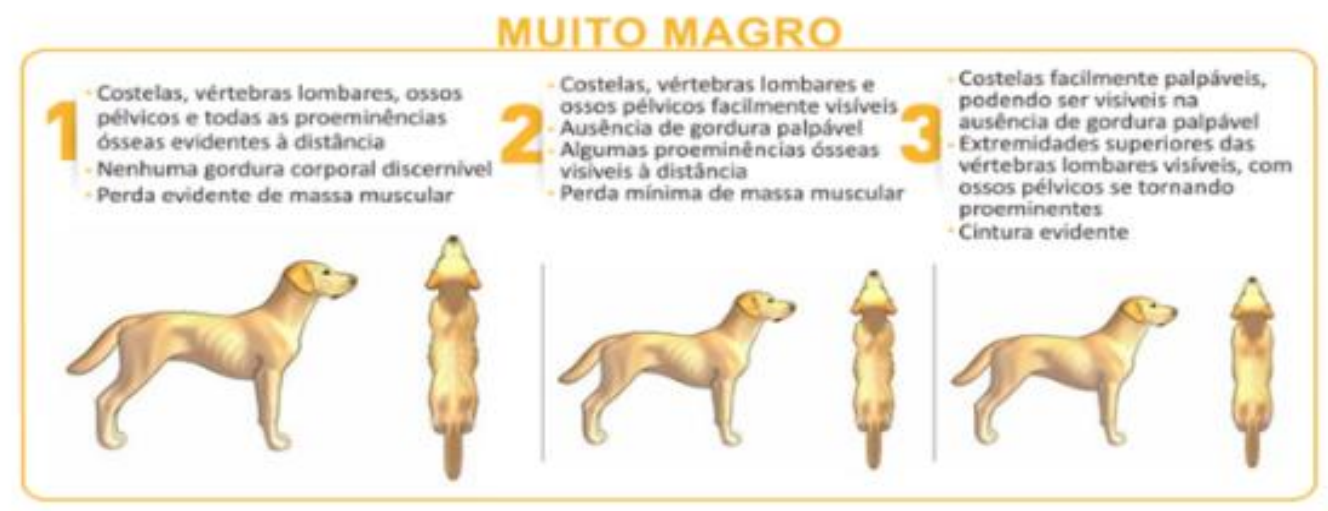

IDEAL

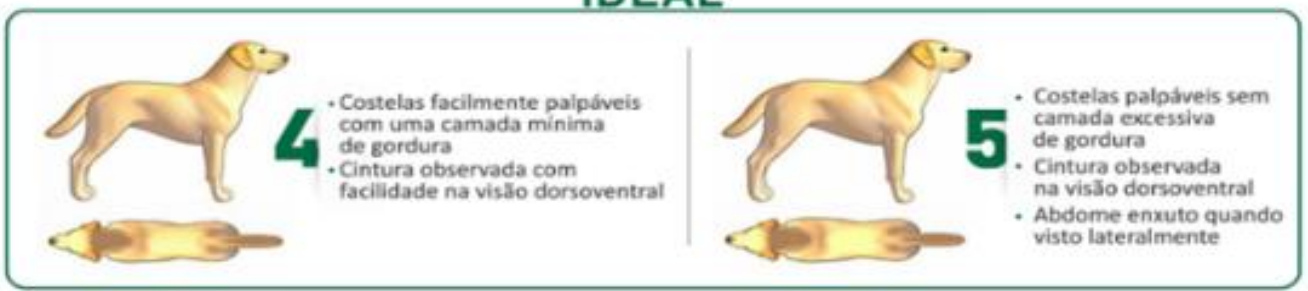

SOBREPESO
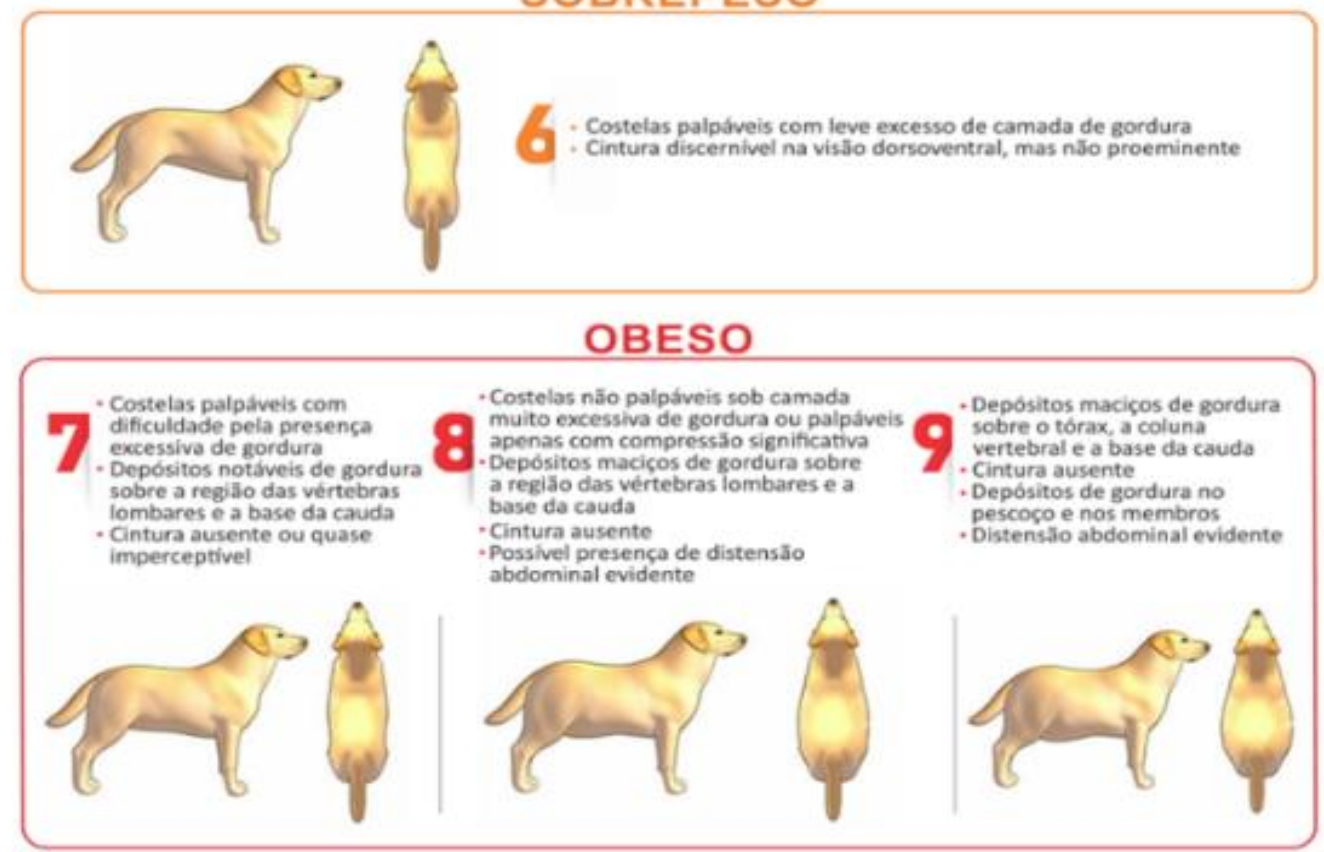

Figura 1. Escore de Condição Corporal, escala de 9 pontos. Fonte: Royal Canin.

\section{Análise e interpretação dos dados}

Os dados foram tabulados no software Excel ${ }^{\circledR}$ para análise. A escolha do melhor método estatístico foi feita após análise prévia dos resultados. Foram estimados a quantidade de animais e os fatores predisponentes da obesidade canina. Foram levados em consideração as diferentes raças e seu peso, e respectivamente, os índices de condição corporal. 


\section{Resultados e discussão}

Participaram da pesquisa 23 tutores com idade entre 18 e 60 anos, variando o grau de instrução (ensino médio incompleto ou completo, superior incompleto ou completo e pós-graduação) e renda familiar mensal (menos de um salário mínimo até seis a dez salários mínimos). A grande maioria fazia uso da televisão e da internet nos tempos livres e para se manter informados.

Os entrevistados responderam essas informações abaixo, referentes ao sexo e a realização ou não dos procedimentos de Orquiectomia e Ovariosalpingohisterectomia dos seus cães (Tabela 1).

Tabela 1. Sexo e apresentação dos cães em castrados e inteiros.

\begin{tabular}{ccccc}
\hline & Machos & & & Fêmeas \\
& 11 & & & 14 \\
\hline Castrados & & Inteiros & Castradas & Inteiras \\
8 & 3 & 8 & 6 \\
\hline
\end{tabular}

A maioria se alimentava de ração seca e comidas diversas, e algumas vezes, com petiscos. A frequência de alimentação desses cães variava entre duas a três vezes ao dia, e à vontade.

Por definição, um cão obeso apresenta um acúmulo de gordura responsável pelo aumento do peso em pelo menos 15\%, em relação ao seu peso ideal (Aptekmann et al., 2014). Na espécie canina, é difícil definir um peso ideal, uma vez que este foi estabelecido apenas para os cães de raça. Este foi um problema encontrado durante a pesquisa, pois a maioria dos cães era sem raça definida. De acordo com Case et al. (1997) para que se determine o aumento excessivo de peso, deve-se comparar o peso atual com pesos prévios ou pesos registrados logo após o animal atingir a idade adulta. Dessa forma, foi possível se basear, principalmente, na observação do formato do corpo do animal, da cintura, das costelas e dos ossos pélvicos, e na palpação das costelas, ou seja, costelas palpáveis, mas não visíveis, representam um peso ideal, costelas dificilmente palpáveis, mostram um excesso de peso, e costelas não palpáveis, concluem que o animal é obeso (Royal Canin Brasil, 2019).

Ao final da entrevista, os 23 tutores responderam que achavam seu animal normal em relação ao estado corporal, relataram que não praticavam atividades físicas ou dietas específicas, e após receberem a informação de que seus animais eram obesos disseram que não sabiam que a obesidade é prejudicial ao animal.

Outro problema observado foi a dificuldade do tutor em perceber o real estado do seu animal e entender que os cães comem menos do que nós (Andrade \& Nascimento, 2005). A maioria dos entrevistados fornecem variedades de comidas e rações à vontade, sem estabelecer um horário específico para fornecer o alimento e sem verificar a quantidade diária ideal para o seu animal, que depende do tamanho, raça e nível de atividade diária. Segundo Oliveira et al. (2010) seria necessária uma dieta equilibrada e moderada, associada a exercícios regulares para esses cães acima do peso. A fim de que o emagrecimento seja efetivo e ocorra de forma saudável, ou seja, lento e gradual, o animal deve ser acompanhado pelo médico veterinário, que determinará metas de perda de peso e orientará o tutor quanto às alterações que deverão ocorrer na rotina do animal.

Na tabela 2 estão apresentadas as demonstrações do nome, idade, raça e escore de condição corporal dos cães participantes da pesquisa.

Cães de meia idade apresentaram maior aumento de peso, e estes foram os mais observados durante a pesquisa. Segundo Fazenda (2010), estes animais, naturalmente, têm um metabolismo mais lento, dormem com maior frequência e se exercitam menos por diversos motivos, tais como: a própria idade; doenças cardíacas ou respiratórias, que diminuem a disposição do animal; artroses, hérnias de disco, entre outros problemas ortopédicos que causam dor e limitam a movimentação. Câncer, doenças hepáticas ou renais, ou outros problemas crônicos também fazem com que o cão não se sinta muito bem, e, portanto, indisposto a brincar, correr ou se exercitar. A Síndrome de Cushing, o hipotireoidismo e outras desordens hormonais podem tornar o metabolismo ainda mais lento, e ainda aumentar o apetite do cão, favorecendo o acúmulo de gorduras. Por fim, o fornecimento de petiscos desordenadamente, a mudança de dieta, de cães adultos para cães idosos, e da oferta de alimentos que causam acúmulos ao longo da vida, são práticas a serem consideradas. 
Tabela 2. Nome, idade, raça e escore de condição corporal dos cães.

\begin{tabular}{|c|c|c|c|}
\hline Nome & Idade & Raça & Escore de condição corporal \\
\hline$\overline{\text { Bud }}$ & 3 anos & Sem raça definida & 5 \\
\hline Farinha & Não soube informar & Sem raça definida & 5 \\
\hline Bóris & 6 anos & Basset Hound & 6 \\
\hline Charlotte & Não soube informar & Sem raça definida & 6 \\
\hline Dogder & 12 anos & Sem raça definida & 6 \\
\hline Duda & 8 anos & Sem raça definida & 6 \\
\hline Lica & 8 anos & Sem raça definida & 6 \\
\hline Pipoca & 1 ano e 2 meses & Sem raça definida & 6 \\
\hline Preta & 10 anos & Sem raça definida & 6 \\
\hline Thor & Não soube informar & Shih Tzu & 6 \\
\hline Timão & 8 anos & Sem raça definida & 6 \\
\hline Luna & 8 anos & Sem raça definida & $6-7$ \\
\hline Fofucho & Não soube informar & Sem raça definida & 7 \\
\hline Lindinha & 5 anos & Pinscher & 7 \\
\hline Nasha & 8 anos & Maltês & 7 \\
\hline Neguinha & 9 anos & Sem raça definida & 7 \\
\hline Pandora & 2 anos e 6 meses & Pug & 7 \\
\hline Théo & 1 ano e 6 meses & Sem raça definida & 7 \\
\hline Trovão & 3 anos & Sem raça definida & 7 \\
\hline Dara & 9 anos & Sem raça definida & 8 \\
\hline Gregório & 13 anos & Lhasa Apso & 8 \\
\hline Hansk & 15 anos & Sem raça definida & 8 \\
\hline Lessie & 11 anos & Labrador & 8 \\
\hline Pequenina & 5 anos & Sem raça definida & 8 \\
\hline Raica & 9 anos & Chow-Chow & 8 \\
\hline
\end{tabular}

Cães obesos, com elevado Escore de Condição Corporal, sofrem mais com o calor, e podem ter dificuldades respiratórias. Esses animais também se cansam facilmente, sentem dores ao caminhar, devido ao grande esforço despendido. O sobrepeso prejudica as articulações e as cartilagens, aumentando a rigidez articular, o que causa ainda mais dor e sofrimento a médio e longo prazo (Toldrá et al., 2012).

Atualmente, a obesidade acomete cerca de $30 \%$ dos cães, mas o problema em si não é apenas estético. A obesidade é reconhecida como uma doença grave, com múltiplas consequências sobre a saúde do animal, e se essas decorrências não forem tratadas corretamente, elas podem diminuir o tempo e qualidade de vida dos cães. Por isso, o tutor precisa estar atento ao seu animal, percebendo que ele sente desconfortos ao fazer atividades diárias ou para se locomover, deve procurar um médico veterinário.

É de grande importância fazer a identificação dos fatores nutricionais e ambientais, para que se possam eliminar as más práticas e os maus hábitos (refeições diárias excessivas e atividade física insuficiente), avaliar as necessidades energéticas, fornece um alimento adequado para cada exigência nutricional, pesar e examinar o seu cão, a fim de expectar um peso corporal saudável e manter o seu animal dentro do Escore de Condição Corporal ideal.

\section{Referências bibliográficas}

Andrade, R. M., \& Nascimento, J. S. (2005). Presença de fungos filamentosos em ração para cães comercializadas na cidade de Pelotas-RS. Arquivo Do Instituto Biológico de São Paulo, 71, 10-12.

Aptekmann, K. P., Suhett, W. G., Junior, A. F. M., Souza, G. B., Tristão, A. P. P. A., Adams, F. K., Aoki, C. G., Junior, R. J. G. P., Carciofi, A. C., \& Tinucci-Costa, M. (2014). Aspectos nutricionais e ambientais da obesidade canina. Ciência Rural, 44(11), 2039-2044. DOI: https://doi.org/10.1590/0103-8478cr20130524

Armstrong, P. J., Yamka, R. M., \& MS, M. B. A. (2008). Obesity's Missing Link: The Union of 
Metabolism, Genome and Disease. Proceedings: Hill's Nutrigenomic Symposium.

Biourges, V. (1997). Obesidade. In Centro de pesquisa e desenvolvimento da Royal Canin.

Brunetto, M. A., Nogueira, S., Sá, F. C., Peixoto, M., Vasconcellos, R. S., Ferraudo, A. J., \& Carciofi, A. C. (2011). Correspondência entre obesidade e hiperlipidemia em cães. Ciência Rural, 41(2), 266271. DOI: https://doi.org/10.1590/s0103-84782011005000004

Burkholder, W. J., \& Toll, P. W. (2000). Obesity. In M. S. Hand (Ed.), Small animal of clinical nutrition (pp. 401-430). Mark Morris Institute.

Carciofi, A. C. (2005). Obesidade e suas conseqüências metabólicas e inflamatórias em cães e gatos.

Case, L. P., Carey, D. P., \& Hirakawa, D. A. (1997). Nutrição canina e felina: manual para profissionais. Harcourt Brace de España.

Diez, M., \& Nguyen, P. (2006). Obesity: epidemiology, pathophysiology and management of the obese dog. Encyclopedia of Canine Clinical Nutrition, 2-53.

Fazenda, M. I. N. (2010). Estudo da relação entre a obesidade e a hipertensão em cães. In Faculdade de Medicina Veterinária: Vol. Master of. Universidade Técnica de Lisboa.

Feitosa, M. L., Zanini, S. F., de Sousa, D. R., Carraro, T. C. L., \& Colnago, L. G. (2015). Fontes amiláceas como estratégia alimentar de controle da obesidade em cães. Ciência Rural, 45(3), 546551. DOI: https://doi.org/10.1590/0103-8478cr20140651

Fleeman, L. M., Seton, E. J., \& Rand, J. (2006). Como abordar: O manejo da obesidade em cães e gatos. Veterinary Focus, 16, 9-15.

German, A. J. (2006). The growing problem of obesity in dogs and cats. The Journal of Nutrition, 136(7), 1940S-1946S. DOI: https://doi.org/10.1093/jn/136.7.1940s

Guimarães, A. L. N., \& Tudury, E. A. (2006). Etiologias, consequiências e tratamentos de obesidades em cães e gatos-revisão. Veterinária Notícias, 12(1), 29-41.

Laflamme, D. P. (2006). Understanding and managing obesity in dogs and cats. Veterinary Clinics of North America: Small Animal Practice, 36(6), 1283-1295. DOI: https://doi.org/10.1016/j.cvsm.2006.08.005

Lazzarotto, J. J. (1999). Relação entre aspectos nutricionais e obesidade em pequenos animais. Revista Da Universidade de Alfenas, 5, 33-35.

Mancini, M. C. (2001). Obstáculos diagnósticos e desafios terapêuticos no paciente obeso. Arquivos Brasileiros de Endocrinologia \& Metabologia, 45(6), 584-608. DOI: https://doi.org/10.1590/s000427302001000600013

Morgante, M. (1999). Obesità negli animali de compagnia: problema emergente. Praxis Veterinaria, $10(2), 18-22$.

Oliveira, M. C., Nascimento, B. C. L., \& Amaral, R. W. C. (2010). Obesidade em cães e seus efeitos em biomarcadores sanguíneos-revisão de literatura. PUBVET, 4, Art. 795-801.

Rodrigues, L. F. (2011). Métodos de avaliação da condição corporal em cães. In Universidade Federal de Goiás.

Royal Canin Brasil. Porque o cão se torna obeso? 2019. Disponível em: http://www.royalcanindobrasil.com.br/pet-news/por-que-o-cao-se-torna-obeso/. Acesso em: 21 jun. 2019.

Salve, M. G. C. (2006). Obesidade e peso corporal: riscos e consequências. Movimento \& Percepção, $6(8), 29-48$.

Silva, P. B. (2014). Perfil e hábitos alimentares de cães em Florianópolis: Vol. GBraduação.

Toldrá, F., Aristoy, M. C., Mora, L., \& Reig, M. (2012). Innovations in value-addition of edible meat by-products. Meat Science, 92(3), 290-296. DOI: http://dx.doi.org/10.1016/j.meatsci.2012.04.004

Vigoureux, R. (2014). Energia e obesidade: processos clínicos associados. Medicina Veterinária, 9(5), $332-336$.

Zoran, D. L. (2010). Obesity in dogs and cats: a metabolic and endocrine disorder. Veterinary Clinics: Small Animal Practice, 40(2), 221-239. DOI: https://doi.org/10.1016/j.cvsm.2009.10.009 